\title{
Retrograde perfusion through superior vena cava reaches the brain during circulatory arrest
}

\author{
Mario Gaudino ${ }^{1}$, Natalia Ivascu ${ }^{2}$, Melissa Cushing ${ }^{3}$, Christopher Lau ${ }^{1}$, Ivancarmine Gambardella ${ }^{1}$, \\ Antonino Di Franco ${ }^{1}$, Lucas B. Ohmes ${ }^{1}$, Monica Munjal ${ }^{1}$, Leonard N. Girardi ${ }^{1}$ \\ ${ }^{1}$ Department of Cardio-Thoracic Surgery, ${ }^{2}$ Department of Anesthesiology, ${ }^{3}$ Clinical Laboratories, Weill-Cornell University, New York, NY, USA \\ Contributions: (I) Conception and design: M Gaudino, N Ivascu; (II) Administrative support: M Gaudino, N Ivascu; (III) Provision of study \\ materials or patients: M Gaudino, N Ivascu, I Gambardella, LN Girardi; (IV) Collection and assembly of data: M Cushing, A Di Franco, C Lau, I \\ Gambardella, LB Ohmes; (V) Data analysis and interpretation: M Gaudino, N Ivascu, LN Girardi; (VI) Manuscript writing: All authors; (VII) Final \\ approval of manuscript: All authors. \\ Correspondence to: Mario Gaudino, MD. Department of Cardiothoracic Surgery, Weill Cornell Medical College, 525 E 68th St, New York, NY 10065, \\ USA. Email: mfg9004@med.cornell.edu.
}

Background: The optimal technique for brain perfusion during circulatory arrest remains controversial. Concern exists that retrograde cerebral perfusion (RCP) via the superior vena cava (SVC) is unable to perfuse the brain. We evaluated whether RCP blood circulates through the brain parenchyma in humans during deep hypothermic circulatory arrest (DHCA). We hypothesized that a significant difference in the levels of S-100 $\beta$ (a protein with very high neuro-sensitivity) between the blood infused in the SVC and the effluent blood returning in the left carotid artery (CA) during RCP, should be regarded as a sign of the circulation of RCP blood through the brain parenchyma.

Methods: We enrolled 10 non-consecutive patients undergoing elective arch-surgery using DHCA and RCP. Circulating S-100 $\beta$ levels were measured at baseline and immediately before DHCA. During DHCA and RCP the difference in S-100 $\beta$ between the SVC and the CA was evaluated after 10 minutes of arrest and immediately before resumption of the circulation. S-100 $\beta$ levels were evaluated using enzyme-linked immunosorbent assay (ELISA).

Results: Mean DHCA duration was $22.4 \pm 7.9$ minutes. Mean S-100 $\beta$ level at baseline was $92.5 \pm 54.9 \mu \mathrm{g} / \mathrm{L}$. After 10 minutes of DHCA the level of S-100ß in the CA was significantly higher than in the SVC (936.9 \pm 326.3 vs. $810.9 \pm 307.4 \mu \mathrm{g} / \mathrm{L}, \mathrm{P}=0.0021)$. This difference was enhanced at the second DHCA sample $(1113.8 \pm 334.2$ vs. $920.5 \pm 340.0 \mu \mathrm{g} / \mathrm{L}, \mathrm{P}=0.0002)$. There was a statistically significant correlation between the duration of DHCA and the percent difference in S-100 $\beta$ level between the SVC and the CA (Pearson's correlation coefficient $=0.902$ ).

Conclusions: RCP is able to perfuse the brain parenchyma in humans during DHCA.

Keywords: Retrograde cerebral perfusion (RCP); deep hypothermic circulatory arrest (DHCA); S-100 $\beta$

Submitted Sep 07, 2017. Accepted for publication Jan 22, 2018.

doi: $10.21037 /$ jtd.2018.01.166

View this article at: http://dx.doi.org/10.21037/jtd.2018.01.166

\section{Introduction}

Methods for cerebral protection during aortic arch surgery are still a matter of debate. Deep hypothermia has traditionally been used to minimize cerebral energy demand and allow a limited period of circulatory arrest deep hypothermic circulatory arrest (DHCA). Major breakthroughs were obtained through the application of selective brain perfusion techniques during the phase of circulatory arrest, which include either anterograde perfusion through the carotid arteries (CA) selective antegrade perfusion (SAP), or retrograde perfusion via the 
Table 1 Preoperative patients' demographics

\begin{tabular}{lc}
\hline Characteristics & Data \\
\hline Male/female & $4 / 6$ \\
Mean age (years) & $68 \pm 15$ \\
Smoking history & 4 \\
Hypertension & 10 \\
Chronic pulmonary disease & 3 \\
Previous stroke & 1 \\
Mean aneurism diameter (cm) & $5.8 \pm 0.6$ \\
Left ventricular ejection fraction (\%) & $48.8 \pm 4.8$ \\
\hline
\end{tabular}

superior vena cava (SVC) retrograde cerebral perfusion (RCP) (1-4). RCP was the first technique to be introduced and has the major advantage of both safety and simplicity. Using this method, blood is directed retrogradely to the brain through the SVC and no manipulation of the epiaortic vessels is required. On the other hand, SAP, although more physiologically appealing, requires cannulation and snaring of one or more of the epi-aortic branches and has the potential for dissection or embolization, especially in patients with atherosclerotic aneurysm or acute aortic dissection.

After an initial phase of almost universal adoption of RCP, most surgical groups shifted to the more complex SAP. Two recent surveys showed that currently in both Europe and Japan less than a third of the aortic centers use RCP $(5,6)$. This shift in neuroprotection was essentially due to concerns of the ability of RCP to provide sufficient brain perfusion. In fact, studies in animal models suggested that RCP was unable to perfuse the brain and that RCP blood reaches the arterial system via arterio-venous shunts, without passing through the cerebral parenchyma (7).

This protocol was conceived as a proof-of concept study to verify that RCP provides effective brain perfusion during DHCA by using a well-known neurochemical marker, the S-100 protein (8). S-100 is an intracellular calcium binding protein with $\alpha$ and $\beta$ subunits, where the $\beta$ subunit is highly brain specific. The $\beta-\beta$ units are present in glial and Schwann cells, whereas the $\alpha-\beta$ subunits appear in glial cells only (8). Even though the role of S-100 $\beta$ as a marker of brain damage has been repeatedly questioned (9), increased S-100 $\beta$ levels in both serum and cerebrospinal fluid have been reported after different types of brain trauma and during DHCA (10).

In this study, S-100 $\beta$ has not been used as a marker of brain damage. Instead, the very high neuro-specificity of the protein, gave us the ability to use $S-100 \beta$ to establish whether the blood that flows retrogradely from the epiaortic vessels during RCP has passed through the brain parenchyma.

We hypothesized that a significant difference in S-100 $\beta$ levels between the blood infused in the SVC and the effluent blood returning in the CA during RCP, should be regarded as a sign of the circulation of RCP blood through the brain parenchyma.

\section{Methods}

This study protocol complies with the principles laid down in the Declaration of Helsinki and was approved by the local Institutional Review Board [2016] (IRB ID: 1505016218). Written informed consent was obtained from every participating patient.

We enrolled 10 non-consecutive patients undergoing elective arch surgery using DHCA and RCP. Enrollment was based on the willingness of the patients to participate to the study and on the availability of the necessary personnel.

Surgery was performed in standard fashion, according to our described technique (11). DHCA was initiated at tympanic temperature of $18{ }^{\circ} \mathrm{C}$. During the entire period of arrest RCP was administered through the SVC cannula after snaring of both venae cavae at rate of $150-300 \mathrm{~mL} / \mathrm{min}$ at $14^{\circ} \mathrm{C}$, keeping the central venous pressure $\leq 30 \mathrm{mmHg}$.

Circulating S-100 $\beta$ levels were measured at baseline (baseline samples were always collected before skin incision from a peripheral venous line) and immediately before DHCA. During DHCA and RCP samples for S-100 $\beta$ analysis were taken simultaneously from the SVC and the left CA after 10 minutes of arrest and immediately before resumption of the circulation.

S-100 $\beta$ sampling was performed using the $\mathrm{CanAg}^{\circledR}$ S-100 $\beta$ Enzyme Immunoassay, a quantitative enzyme-linked immunosorbent assay (ELISA). The coefficient of variation of this assay is less than or equal to $6.3 \%$ (12).

Data are expressed as mean \pm standard deviation and median if appropriate. Comparison between S-100 $\beta$ levels in the SVC and the left CA was performed with paired, 2-tailed $t$-test. Pearson's coefficient correlation was used to explore the association between S-100 $\beta$ levels and time of DHCA.

\section{Results}

Details of the ten patients enrolled in the study are 


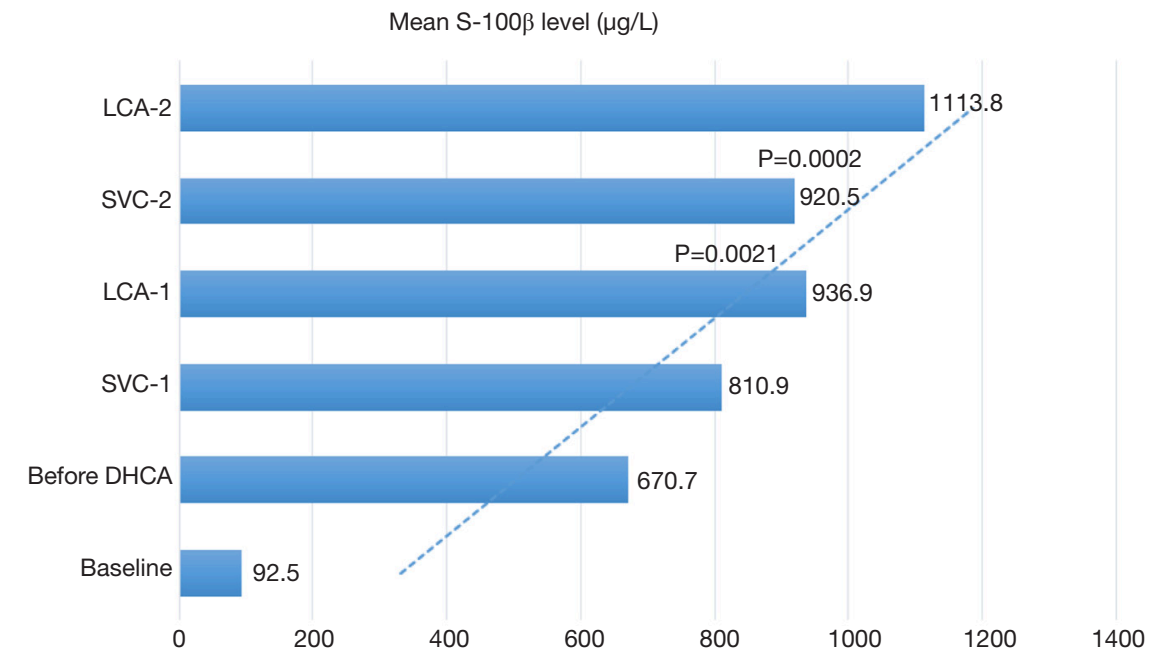

Figure 1 Results of S-100 $\beta$ assay. DHCA, deep hypothermic circulatory arrest; SVC-1, superior vena cava after 10 minutes of circulatory arrest; SVC-2, superior vena cava immediately before end of circulatory arrest; LCA-1, left carotid artery after 10 minutes of circulatory arrest; LCA-2, left carotid artery immediately before end of circulatory arrest.

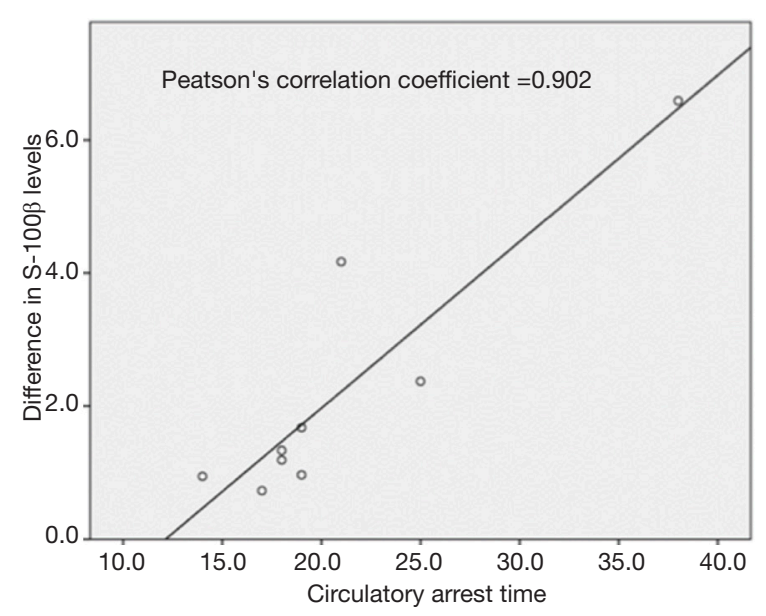

Figure 2 Pearson's coefficient correlation between the duration of circulatory arrest and the percent difference in S-100 $\beta$ level between the superior vena cava and the left carotid artery.

summarized in Table 1.

Most of the cases were women in their seventh decade. Hypertension and smoking were the most prevalent vascular risk factors. Mean aneurysm size at the time of surgery was $5.8 \mathrm{~cm}$.

Eight patients underwent hemiarch replacement and two had total arch replacement. In one patient, the arch procedure was a redo operation and another case had concomitant aortic root replacement. Mean cardiopulmonary bypass time was $133.1 \pm 22.8$ minutes and DHCA duration was $22.4 \pm 7.9$ minutes (median 19 minutes).

There was no operative mortality. One patient suffered a postoperative stroke. The in-hospital course was unremarkable for the remaining 9 cases.

Results of the S-100 $\beta$ sampling are summarized in Figure 1. The mean S-100ß level at baseline was $92.5 \pm 54.9 \mu \mathrm{g} / \mathrm{L}$. Immediately before DHCA mean circulating S-100 $\beta$ level was $670.7 \pm 355.2 \mu \mathrm{g} / \mathrm{L}$. After 10 minutes of DHCA the level of $\mathrm{S}-100 \beta$ in the left CA was significantly higher than in the SVC $(936.9 \pm 326.3$ vs. $810.9 \pm 307.4 \mu \mathrm{g} / \mathrm{L}, \mathrm{P}=0.0021)$. This difference was enhanced at the second DHCA sample $(1113.8 \pm 334.2$ vs. $920.5 \pm 340.0 \mu \mathrm{g} / \mathrm{L}, \mathrm{P}=0.0002)$. There was a statistically significant correlation between the duration of DHCA and the percent difference in S-100 $\beta$ level between the SVC and the left CA (Pearson's correlation coefficient $=0.902$, see Figure 2).

\section{Discussion}

RCP was the first method of cerebral perfusion adopted during operations on the aortic arch in DHCA. Since the seminal paper of Ueda (4), several authors (including us) have used RCP with excellent clinical results. Estrera et al. used RCP as the main brain protective strategy in 1,107 patients undergoing aortic arch repair and reported a $2.8 \%$ incidence of permanent neurological deficit (13). We have previously published a series of 879 consecutive aortic arch operations using DHCA and RCP with an incidence of 
stroke of $1.4 \%$ (11).

However, in recent years RCP has been progressively abandoned in favor of the more complex SAP. Recent surveys showed that only a third of aortic centers in Europe and Japan currently use RCP for elective arch surgery $(5,6)$.

The progressive abandonment of RCP arose mainly from the concern based on animal studies (7) that RCP blood reaches the arterial system via arterio-venous shunts without passing through the cerebral parenchyma and that RCP is thus unable to provide effective brain perfusion. The results of experimental and clinical data on the subject have been conflicting. Cheung and associates measured $\mathrm{O}_{2}$ extraction, $\mathrm{pH}$, and $\mathrm{PCO}_{2}$ from the RCP inflow and outflow in humans which demonstrated near maximal brain oxygen extraction suggesting relative cerebral hypoperfusion during RCP (14). A cadaveric study using in vitro venous retroperfusion showed that in humans most of the valves of the internal jugular vein are competent and potentially obstruct RCP (15). Transcranial Doppler studies aimed at evaluating the flow velocity in the middle cerebral artery during RCP gave mixed results (16). On the other hand, Wong and Bonser coupling metabolic and Doppler studies found reversal of brain blood flow during RCP in 10 patients (17). Pagano and colleagues, in an elegant study using Technetium 99 brain perfusion scan, were able to show cerebral flow during RCP in three patients (18). More recently, Endo and coauthors using a modified RCP technique and a retinal camera reported flow in the retinal vessels during RCP in 8 cases of total arch replacement (19).

Several animal models used different approaches and methodologies to investigate the subject. The results were again mixed with some experiments supporting the efficacy of RCP and others denying it (20-23).

We decided to use S-100 $\beta$, a protein with very high neuro-sensitivity, as a marker of circulation in the cerebral parenchyma. We did not use $\mathrm{S}-100 \beta$ as a marker of brain damage, as this has been repeatedly questioned (9). However, the circulating level of S-100 $\beta$ has been shown to progressively increase during DHCA (10). As S-100 $\beta$ is specific to the brain (8), we hypothesized that a significant difference in S-100 $\beta$ levels between the blood infused in the SVC and the effluent blood returning in the left CA during RCP, should be regarded as a sign of the circulation of RCP blood through the brain parenchyma.

We found that the level of S-100 $\beta$ in the left CA was always significantly higher than in the SVC and that there was a statistically significant correlation between the duration of DHCA and the percent difference in S-100 $\beta$ level (Pearson's correlation coefficient $=0.902$, see Figures 1 and 2).

These results strongly suggest that RCP is able to perfuse the brain parenchyma in humans during DHCA.

Our findings provide the physio-pathological background to the clinical observation that RCP is an effective brain protection strategy in patients undergoing operations in DHCA. In fact, two recent meta-analysis on the comparison of the neurological outcomes of RCP vs. antegrade perfusion were unable to prove any clinically significant difference between the two techniques $(24,25)$.

This study was designed as a simple proof of concept. We did not aim at evaluating the adequacy of RCP to the cerebral oxygen demand nor at evaluating the efficacy of the different protocols for performing RCP. Further extensive investigation on this subject is required.

Also, even though we paid great attention in sampling effluent blood well within the left CA, the possibility of a contamination from extracranial sources cannot be ruled out completely. However, as S-100 $\beta$ is produced almost exclusively in the brain, dilution due to blood from noncerebral regions would have led to an underestimation and not an overestimation of cerebral perfusion during RCP.

\section{Conclusions}

In conclusion, this study demonstrates that RCP provides brain perfusion in humans undergoing DHCA. Further studies on the adequacy of RCP flow to cerebral oxygen demand and on the most appropriate technique to perform $\mathrm{RCP}$ are required.

\section{Acknowledgements}

Funding: This project was supported by NIH/National Center for Advancing Translational Sciences (NCATS) Grant \#UL1TR000457 through the Clinical and Translational Science Center of Cornell University.

\section{Footnote}

Conflicts of Interest: The authors have no conflicts of interest to declare.

Ethical Statement: This study protocol complies with the principles laid down in the Declaration of Helsinki and was approved by the local Institutional Review Board [2016] 
(IRB ID: 1505016218). Written informed consent was obtained from every participating patient.

\section{References}

1. Bachet J, Guilmet D, Goudot B, et al. Cold cerebroplegia. A new technique of cerebral protection during operations on the transverse aortic arch. J Thorac Cardiovasc Surg 1991;102:85-93; discussion 93-4.

2. Frist $\mathrm{WH}$, Baldwin JC, Starnes VA, et al. A reconsideration of cerebral perfusion in aortic arch replacement. Ann Thorac Surg 1986;42:273-81.

3. Kazui T, Inoue N, Yamada O, et al. Selective cerebral perfusion during operation for aneurysms of the aortic arch: a reassessment. Ann Thorac Surg 1992;53:109-14.

4. Ueda Y, Miki S. Retrograde cerebral perfusion. Ann Thorac Surg 1992;53:364-5.

5. De Paulis R, Czerny M, Weltert L, et al. Current trends in cannulation and neuroprotection during surgery of the aortic arch in Europe. Eur J Cardiothorac Surg 2015;47:917-23.

6. Okita Y, Miyata H, Motomura N, et al. A study of brain protection during total arch replacement comparing antegrade cerebral perfusion versus hypothermic circulatory arrest, with or without retrograde cerebral perfusion: analysis based on the Japan Adult Cardiovascular Surgery Database. J Thorac Cardiovasc Surg 2015;149:S65-73.

7. Ehrlich MP, Hagl C, McCullough JN, et al. Retrograde cerebral perfusion provides negligible flow through brain capillaries in the pig. J Thorac Cardiovasc Surg 2001;122:331-8.

8. Kligman D, Hilt DC. The S100 protein family. Trends Biochem Sci 1988;13:437-43.

9. Olivecrona M, Rodling-Wahlström M, Naredi S, et al. S-100B and neuron specific enolase are poor outcome predictors in severe traumatic brain injury treated by an intracranial pressure targeted therapy. J Neurol Neurosurg Psychiatry 2009;80:1241-7.

10. Astudillo R, Van der Linden J, Radegran K, et al. Elevated serum levels of S-100 after deep hypothermic arrest correlate with duration of circulatory arrest. Eur J Cardiothorac Surg 1996;10:1107-12; discussion 1113.

11. Girardi LN, Shavladze N, Sedrakyan A, et al. Safety and efficacy of retrograde cerebral perfusion as an adjunct for cerebral protection during surgery on the aortic arch. J Thorac Cardiovasc Surg 2014;148:2927-33.

12. Erickson JA, Grenache DG. Comparison of three assays for quantifying S-100B in serum. Clin Chim Acta 2011;412:2122-7.

13. Estrera AL, Miller CC 3rd, Lee TY, et al. Ascending and transverse aortic arch repair: the impact of retrograde cerebral perfusion. Circulation 2008;118:S160-6.

14. Cheung AT, Bavaria JE, Pochettino A, et al. Oxygen delivery during retrograde cerebral perfusion in humans. Anesth Analg 1999;88:8-15.

15. Künzli A, Zingg PO, Zünd G, et al. Does retrograde cerebral perfusion via superior vena cava cannulation protect the brain? Eur J Cardiothorac Surg 2006;30:906-9.

16. Tanoue Y, Tominaga R, Ochiai Y, et al. Comparative study of retrograde and selective cerebral perfusion with transcranial Doppler. Ann Thorac Surg 1999;67:672-5.

17. Wong C, Bonser RS. Retrograde perfusion and true reverse brain blood flow in humans. Eur J Cardiothorac Surg 2000;17:597-601.

18. Pagano D, Boivin CM, Faroqui MH, et al. Surgery of the thoracic aorta with hypothermic circulatory arrest: experience with retrograde perfusion via the superior vena cava and demonstration of cerebral perfusion. Eur J Cardiothorac Surg 1996;10:833-8; discussion 839.

19. Endo H, Ishii H, Tsuchiya H, et al. Observations of retinal vessels during intermittent pressure-augmented retrograde cerebral perfusion in clinical cases. Interact Cardiovasc Thorac Surg 2016;23:259-65.

20. Duebener LF, Hagino I, Schmitt K, et al. Direct visualization of minimal cerebral capillary flow during retrograde cerebral perfusion: an intravital fluorescence microscopy study in pigs. Ann Thorac Surg 2003;75:1288-93.

21. Li Z, Yang L, Summers R, et al. Is maintenance of cerebral hypothermia the principal mechanism by which retrograde cerebral perfusion provides better brain protection than hypothermic circulatory arrest? A study in a porcine model. J Card Surg 2004;19:28-35.

22. Usui A, Hotta T, Hiroura M, et al. Retrograde cerebral perfusion through a superior vena caval cannula protects the brain. Ann Thorac Surg 1992;53:47-53.

23. Ye J, Ryner LN, Kozlowski P, et al. Retrograde cerebral perfusion results in flow distribution abnormalities and neuronal damage. A magnetic resonance imaging and histopathological study in pigs. Circulation 1998;98:II313-8.

24. Guo S, Sun Y, Ji B, et al. Similar cerebral protective effectiveness of antegrade and retrograde cerebral perfusion during deep hypothermic circulatory arrest in aortic surgery: a meta-analysis of 7023 patients. Artif 
Organs 2015;39:300-8.

25. Hu Z, Wang Z, Ren Z, et al. Similar cerebral protective effectiveness of antegrade and retrograde cerebral perfusion combined with deep hypothermia circulatory arrest in aortic arch surgery: a meta-analysis and systematic review of 5060 patients. J Thorac Cardiovasc Surg 2014;148:544-60.

Cite this article as: Gaudino $M$, Ivascu N, Cushing $M$, Lau C, Gambardella I, Di Franco A, Ohmes LB, Munjal M, Girardi LN. Retrograde perfusion through superior vena cava reaches the brain during circulatory arrest. J Thorac Dis 2018;10(3):1563-1568. doi: 10.21037/jtd.2018.01.166 